\title{
Shear-induced deconfinement of hard disks
}

\author{
Nikolai Jahreis $^{1} \cdot$ Matthias Schmidt $^{1}$
}

Received: 20 January 2020 / Revised: 22 March 2020 / Accepted: 23 March 2020 / Published online: 6 May 2020

(C) The Author(s) 2020

\begin{abstract}
Using Brownian dynamics simulations, we investigate the response to shear of a two-dimensional system of quasi-hard disks that are confined in the velocity gradient direction by a smooth external potential. Shearing the confined system leads to a homogenization of the one-body density profile. In order to rationalize this deconfinement effect, we split the internal onebody force field into adiabatic and superadiabatic contributions. We demonstrate that the superadiabatic force field consists of viscous and of structural contributions. We give an empirical scaling law that yields results for the superadiabatic force profiles both in the flow and in the gradient direction, in excellent agreement with the simulation data.
\end{abstract}

Keywords Colloids $\cdot$ Rheology $\cdot$ Power functional theory

\section{Introduction}

Applying shear to a complex substance constitutes a means to drive the system out of equilibrium in a well-controlled way, and results in arguably one of the most fundamental nonequilibrium setups [1, 2]. In its simplest form, shearing is characterized by the shear rate $\dot{\gamma}$ as the only relevant (and scalar) nonequilibrium parameter. The physics at play, even only in steady state, is however fundamentally different from the equilibrium properties of the same material. Hence, shearing is an excellent model situation for systematically studying soft matter out of equilibrium.

For the important material class of colloidal dispersions, Matthias Ballauff and collaborators have performed sterling work, developing and exploiting ingeniously tailored particles that respond to temperature variation. Despite the quite complex internal core-shell structure of these thermosensitive colloids [3-20], the particles interact via an essentially short-ranged steeply repulsive pair potential. Changing the temperature facilitates systematically changing the effective particle size and hence the typical length scale of the interparticle interactions. The underlying mechanism is the thermoresponse of the polymeric particle shell. Controlling the particle size allows to control accordingly the effective

Matthias Schmidt

Matthias.Schmidt@uni-bayreuth.de

1 Theoretische Physik II, Physikalisches Institut, Universität Bayreuth, 95447, Bayreuth, Germany colloidal packing fraction in the system. As the response to shear depends very sensitively on the packing fraction, thermosensitive colloids give direct access to this crucial thermodynamic parameter. The shear rate $\dot{\gamma}$, often expressed as a dimensionless Peclet number Pe [1], is an external parameter that controls the degree of nonequilibrium driving that the system is exposed to.

Mode-coupling theory (MCT), as spearheaded by Fuchs and coworkers [5, 6, 8-12, 15-17, 20], has provided a platform for rationalizing and in many cases quantitatively describing the results from experiments, as obtained rheometrically. Much insightful work is based on the "schematic model" $[5,6,11,15,17]$ of MCT. The impressive degree of consistency, in qualitative and in quantitative terms, for important quantities is achieved with a very moderate level of empirical input. This is remarkable, as the considered quantities vary typically over many orders of magnitude. MCT operates on the level of two-body correlation functions. In particular, via GreenKubo relations, the equilibrium stress-stress autocorrelator is set in relation to the nonequilibrium response under shear. The full theory is hence a complex one, which justifies the need for having the schematic model, which is regarded as describing the essential features of the dynamics of a generic time correlation function, in particular historydependence via a memory integral over previous times. The sole equilibrium input in MCT is the static structure factor $S(q)$ of the fluid. The entirety of the time-dependent nonequilibrium phenomena that occur under driving arises from the dynamical structure of the MCT equations of motion. 
On principal grounds, one could expect that additional equilibrium information, besides $S(q)$, could be required or be at least useful. Brader, Krüger, and their coworkers [21-25] have hence gone beyond the static limitation by incorporating ideas from classical density functional theory (DFT) for inhomogeneous fluids [26, 27]. DFT is a framework that is genuinely adapted for and capable of describing situations where the locally resolved microscopic density distribution $\rho(\mathbf{r})$ is inhomogeneous in space: $\rho(\mathbf{r}) \neq$ const, where $\mathbf{r}$ indicates position. Two important (and useful) relationships for equilibrium systems are the following: (i) The internal contribution to the one-body force profile, $\mathbf{f}_{\mathrm{ad}}(\mathbf{r})$, is solely dependent on the density profile, but it is independent of the external forces that act in the system (say due to the presence of walls). Hence, $\mathbf{f}_{\mathrm{ad}}(\mathbf{r})=\mathbf{f}_{\mathrm{ad}}(\mathbf{r},[\rho])$, where the brackets indicate a functional relationship of mapping the entire function $\rho\left(\mathbf{r}^{\prime}\right)$ at all space points $\mathbf{r}^{\prime}$ to the force at the given position $\mathbf{r}$. The internal force field $\mathbf{f}_{\mathrm{ad}}(\mathbf{r})$ arises from all interparticle forces that the remaining particles exert on a particle located at position $\mathbf{r}$ in an equilibrium situation. (ii) Two-body correlation functions are contained in the approach and are accessible, alternatively, by the Ornstein-Zernike or the test particle routes (see Ref. [28] for a recent account of the virtues of the test particle approach when combined with an approximate free-energy functional).

Krüger and Brader's work [21-25] for systems under shear is based on the so-called dynamical DFT [26, 29, 30] for nonequilibrium dynamics. Nevertheless, the approach described in Refs. [21-25] still genuinely works on the two-body level, which a priori puts high strain both on the physical intuition that is required to devise approximation schemes, as well as on computational demand. Although dynamical DFT operates on the one-body level, for a sheared system with flow direction being orthogonal to the density gradient, the bare DFT [26, 29, 30] gives a null result, in contrast to Krüger and Brader's more sophisticated theory [21-25]. Dynamical DFT, in its bare version [26, $29,30]$, is hence defunct for the description of arguably the most basic nonequilibrium situation of a colloidal system, or more generally, of an inhomogeneous classical liquid. The reason for the failure is of fundamental nature: the true nonequilibrium dynamics is described as consisting of a sequence of "adiabatic states" that are taken to be at equilibrium. Clearly, this assumption is not true in general, and for the case of shear, it is in quite striking contradiction to reality.

However, there is hope for formulating a complete description on the one-body level, as the superadiabatic contributions that occur above the adiabatic effects (correctly accounted for in the dynamical DFT) are both well- defined and well-characterizable objects [31, 32] from an extended, kinematic functional point of view. Here, the microscopically resolved velocity profile $\mathbf{v}(\mathbf{r}, t)$ is a variable on par with the time-dependent density profile $\rho(\mathbf{r}, t)$, where $t$ indicates time. The full nonequilibrium dynamics is hence driven both by adiabatic effects, which are functionally dependent only on the instantaneous density distribution, and superadiabatic effects, which possess kinematic dependence on both $\rho\left(\mathbf{r}, t^{\prime}\right)$ and $\mathbf{v}\left(\mathbf{r}, t^{\prime}\right)$ for times $t^{\prime} \leq$ $t$, where $t$ is the time of interest. Hence, the superadiabatic contribution is nonlocal both in space and in time, while the adiabatic contribution is Markovian (instantaneous) in time, but nonlocal in space.

For the (important) case of the internal one-body force field $\mathbf{f}_{\text {int }}(\mathbf{r}, t)$, which is generated from the underlying interparticle interaction potential, the adiabatic-superadiabatic splitting is

$\mathbf{f}_{\text {int }}(\mathbf{r}, t)=\mathbf{f}_{\mathrm{ad}}(\mathbf{r}, t,[\rho])+\mathbf{f}_{\text {sup }}(\mathbf{r}, t,[\rho, \mathbf{v}])$.

This formal result [31] has implications that are important, practical, and testable. In particular, the independence of $\mathbf{f}_{\text {sup }}(\mathbf{r}, t)$ from the external forces allows to disentangle intrinsic from external effects and hence it offers the potential for deep insights into the coupled nature of the nonequilibrium many-body physics [31].

Power functional theory (PFT) not only provides the existence of the functional map $(\rho, \mathbf{v}) \rightarrow \mathbf{f}_{\text {sup }}$. It also establishes on a microscopic footing a rigorous minimization principle for the velocity profile, or equivalently for the microscopic one-body current $\mathbf{J}(\mathbf{r}, t)=\mathbf{v}(\mathbf{r}, t) \rho(\mathbf{r}, t)$, implying vanishing functional derivative at the minimum, $\delta R_{t}[\rho, \mathbf{J}] / \delta \mathbf{J}(\mathbf{r}, t)=0$. Here, $R_{t}$ is the (total) free power functional, which consists of a sum of ideal, external, adiabatic, and superadiabatic contributions. The latter is the superadiabatic excess (i.e., over ideal) free power functional $P_{t}^{\text {exc }}$. The superadiabatic internal force profile is obtained as a functional derivative $\mathbf{f}_{\text {sup }}(\mathbf{r}, t)=-\delta P_{t}^{\operatorname{exc}} / \delta \mathbf{J}(\mathbf{r}, t)$. As in equilibrium DFT, the adiabatic internal force profile is obtained via $\mathbf{f}_{\text {ad }}(\mathbf{r}, t)=-\nabla \delta F_{\text {exc }} / \delta \rho(\mathbf{r}, t)$, where $F_{\text {exc }}[\rho]$ is the intrinsic excess Helmholtz free-energy functional, and nabla denotes the derivative with respect to position $\mathrm{r}$. PFT has been both applied and extended over a growing range of physical systems and situations [31-53]. We give a brief overview in the following.

Within PFT, the two-body structure [33-37] is accessible via the nonequilibrium Ornstein-Zernike [33, 34] and the dynamical test particle routes [35-37]. Functional integration methods were developed [38], and the relevance of local particle number conservation was demonstrated [56, 57]. Expressing the superadiabatic free power functional via the velocity gradient [39-43] has facilitated the description 
of viscous [39, 40, 42] and of structural superadiabatic force contributions [41-43]. Superadiabatic forces are accessible in many-body simulations [32, 44, 45]. Efficient methods such as force sampling [54] render this a standard task. PFT has been applied to the bulk and interfacial behavior of active Brownian particles [46-50]. Here, the positionand orientation-resolved one-body fields have proven to be appropriate variables. For the case of sedimentation of the active ideal gas, an analytical solution for these fields could be constructed [55]. PFT was extended for the description of inertial classical [51] and of quantum many-body dynamics [52, 53]. The fundamental differences between canonical and grand canonical schemes have been addressed in equilibrium [56] and for the dynamics [57].

Here, we present the first study of superadiabatic forces in a system under simple shear, i.e., flow that is characterized by linear dependence of the velocity on position, and hence spatially constant imposed shear rate $\dot{\gamma}$. In order to trigger a response of the system on the one-body level, as befits the concepts underlying the PFT framework, we expose the sheared two-dimensional system to an additional confining external potential. We choose the (arguably) simplest possible geometry, where the external potential $V_{\text {ext }}(y)$ varies only in the shear gradient direction $y$. As a consequence, all one-body quantities become functions of (only) the $y$-coordinate, and the system remains translationally invariant in the flow direction $x$.

The above setup is interesting, as it allows the study of the influence of shear on a prototypical confined situation for a simple fluid, i.e., that of a periodically varying, oscillating potential (along the $y$-direction of the shear gradient). We can hence monitor both the structural forces that act in the $y$-direction and the viscous forces that act in the flow direction $x$. We present simple phenomenological scaling laws that fit the results quantitatively. We consider moderate fluid densities only and hence deliberately stay away from the intricacies of the glass transition. We do, however, consider cases of high driving, where we find the system to exhibit interesting and apparently universal saturation behavior. The saturation relates the external conservative force $-\nabla V_{\text {ext }}$ and the intrinsic superadiabatic force to each other. This is a remarkable mechanism, as external and superadiabatic forces are very different in origin. As a result of the shear, the density profile homogenizes, and hence, shear acts against the confinement induced by $V_{\text {ext }}$.

This paper is organized as follows: In "Description of the system," the physical system considered is described and the Brownian dynamics (BD) simulation algorithm is laid out. The results of the simulations are presented in "Results." In "Conclusions," we conclude and give an outlook on possible future work.

\section{Description of the system}

\section{Microscopic dynamics}

Our two-dimensional system consists of $N$ (indistinguishable) circular particles suspended in an incompressible implicit solvent. The particles are quasi-hard disks; their pair interaction potential is given by

$\phi(r)=\epsilon\left(\frac{\sigma}{r}\right)^{36}$,

where $r$ is the center-center distance between two particles, $\sigma=$ const denotes the diameter of the particles, and $\epsilon=$ const sets the energy scale.

In addition to their internal interactions, the particles are subject to thermal fluctuations and to an external force field. The thermal fluctuations induce a random force $\mathbf{f}_{i}^{\text {ran }}(t)$ on particle $i$ with the following statistical properties:

$$
\begin{aligned}
\left\langle\mathbf{f}_{i}^{\mathrm{ran}}(t)\right\rangle & =0, \\
\left\langle\mathbf{f}_{i}^{\mathrm{ran}}(t) \mathbf{f}_{j}^{\mathrm{ran}}\left(t^{\prime}\right)\right\rangle & =2 \xi k_{B} T \delta\left(t-t^{\prime}\right) \mathbb{1} \delta_{i j},
\end{aligned}
$$

where the angles denote an average over different realizations of the noise, $t$ and $t^{\prime}$ indicate time arguments, $\xi$ denotes the friction coefficient, $k_{B}$ indicates the Boltzmann constant, $T$ indicates the temperature, $\delta(\cdot)$ represents the Dirac delta distribution, $\mathbb{1}$ is the $2 \times 2$ unit matrix, and the integers $i, j=1 \ldots N$ label the particles.

The external force field splits according to

$\mathbf{f}_{\text {ext }}\left(y_{i}\right)=\mathbf{f}_{\text {shear }}\left(y_{i}\right)-\nabla_{i} V_{\text {ext }}\left(y_{i}\right)$

where $y_{i}$ indicates the $y$-coordinate of particle $i$ and $\nabla_{i}$ is the derivative with respect to the position $\mathbf{r}_{i}$ of particle $i$. Here, the first term is a non-conservative linear shear field acting in the $x$-direction and the second term is a conservative compression force along the $y$-direction, given, respectively, by

$\begin{aligned} \mathbf{f}_{\text {shear }}(y) & =\dot{\gamma} y \hat{\mathbf{e}}_{x}, \\ V_{\text {ext }}(y) & =V_{0}^{\text {ext }}\left(1-\frac{8 y^{2}}{L^{2}}+\frac{16 y^{4}}{L^{4}}\right),\end{aligned}$

where the shear rate $\dot{\gamma}$ is an inverse timescale that characterizes the strength of the driving and $\hat{\mathbf{e}}_{x}$ is the unit vector in the $x$-direction. The confining potential (6) compresses the system symmetrically in the $y$-direction towards $y=L / 2$; the constant $V_{0}^{\text {ext }}$ sets the depth of the potential well. The maximum is at $y=0$, and minimum of the well is at $y= \pm L / 2$, such that $V_{\text {ext }}(0)-V_{\text {ext }}(L / 2)=$ $V_{0}^{\text {ext }}$. Figure 1 shows an illustration of the model. We use periodic boundary conditions in both spatial directions; $L$ denotes the size of the (square) simulation box. 


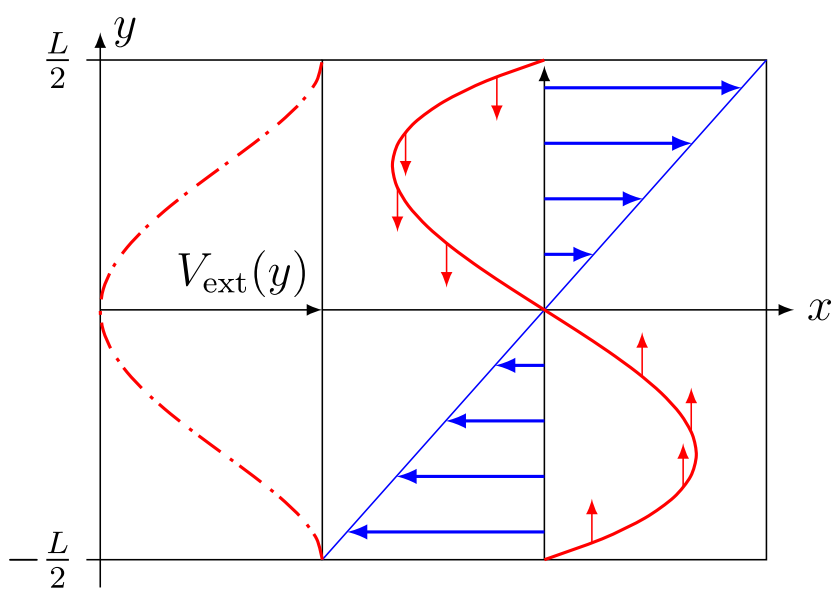

Fig. 1 Sketch of the geometry of the system. Shown are the external potential $V_{\text {ext }}(y)$ that compresses the system towards $y=0$ (dasheddotted red line); the resulting conservative force field $-\nabla V_{\text {ext }}$ acting in the $y$-direction (red arrows indicate direction and the solid red line indicates the magnitude); and non-conservative force field (blue arrows) $\mathbf{f}_{\text {shear }}$ due to the externally imposed shear flow. The system is translationally invariant in the $x$-direction. Lees-Edwards boundary conditions render the shear force continuous in the periodic images of the system along the $y$-direction

We consider overdamped dynamics, where the inertia of the particles can be neglected and the dynamics of the system is described by the Langevin equation of motion [27]:

$$
\begin{aligned}
\xi \dot{\mathbf{r}}_{i}(t) & =\mathbf{f}_{i}^{\mathrm{int}}\left(\mathbf{r}^{N}\right)+\mathbf{f}_{\mathrm{ext}}\left(y_{i}\right)+\mathbf{f}_{i}^{\mathrm{ran}}(t), \\
\mathbf{f}_{i}^{\mathrm{int}}\left(\mathbf{r}^{N}\right) & =-\nabla_{i} \sum_{j=1}^{N} \sum_{k=j+1}^{N} \phi\left(\left|\mathbf{r}_{j}-\mathbf{r}_{k}\right|\right),
\end{aligned}
$$

with $\dot{\mathbf{r}}_{i}$ denoting the time derivative of $\mathbf{r}_{i}$.

\section{One-body correlation functions}

In steady state, the system is invariant under translation in the $x$-direction and all one-body quantities (as described below) only depend on the $y$-coordinate. In our simulations, we check that a steady state is reached before sampling any quantities of interest.

As the one-body density,

$\rho(\mathbf{r})=\left\langle\sum_{i=1}^{N} \delta\left(\mathbf{r}-\mathbf{r}_{i}\right)\right\rangle$,

will not vary in time in steady state, its time derivative $\partial \rho(\mathbf{r}) / \partial t=0$, and the continuity equation reduces to

$$
\nabla \cdot \mathbf{J}=0
$$

implying that the particle current $\mathbf{J}(y)$ is also constant in time. Here, the one-body current is defined as

$\mathbf{J}(\mathbf{r})=\left\langle\sum_{i=1}^{N} \delta\left(\mathbf{r}-\mathbf{r}_{i}\right) \mathbf{v}_{i}\right\rangle$,

where the velocity $\mathbf{v}_{i}$ of particle $i$ at time $t$ is given by a centered difference of its position vector [45],

$\mathbf{v}_{i}(t)=\frac{\mathbf{r}_{i}(t+\Delta t)-\mathbf{r}_{i}(t-\Delta t)}{2 \Delta t}$,

where $\Delta t$ is the time step of the numerical integration routine (as detailed in "Brownian dynamics simulations" below). See Ref. [45] for the derivation of the finite difference expression (12) for the velocity in Brownian dynamics. We split the current into three distinct contributions, corresponding to the force densities due to internal interactions $\left(\mathbf{F}_{\text {int }}\right)$, thermal diffusion $\left(-k_{B} T \nabla \rho\right)$, and external influence $\left(\rho \mathbf{f}_{\mathrm{ext}}\right)$, and hence,

$$
\begin{aligned}
\xi \mathbf{J} & =\mathbf{F}_{\text {int }}-k_{B} T \nabla \rho+\rho \mathbf{f}_{\text {ext }} \\
& =\rho\left(\mathbf{f}_{\text {int }}+\mathbf{f}_{\text {ran }}+\mathbf{f}_{\text {ext }}\right) .
\end{aligned}
$$

Here, the one-body internal force density distribution is defined as

$\mathbf{F}_{\text {int }}(\mathbf{r})=\left\langle\sum_{i=1}^{N} \delta\left(\mathbf{r}-\mathbf{r}_{i}\right) \mathbf{f}_{i}^{\text {int }}\right\rangle$,

where $\mathbf{f}_{i}^{\text {int }}$, as given by Eq. 8, is the force acting on particle $i$ due to the internal interactions with all other particles in the system. The force fields are obtained by dividing force densities by the density profile, i.e., $\mathbf{f}_{\text {int }}=\mathbf{F}_{\text {int }} / \rho$ and $\mathbf{f}_{\mathrm{ran}}=-k_{B} T(\nabla \rho) / \rho \equiv-k_{B} T \nabla \ln \rho$. In the results described below, we present data for force fields rather than for force densities. The thermal fluctuations generate an average force caused by the thermal fluctuations,

$\mathbf{f}_{\mathrm{ran}}(y)=-k_{B} T \nabla \ln \rho$.

Splitting the current and therefore the forces is useful as it enables us to perform the splitting of the internal force field into its adiabatic and super adiabatic contribution. The velocity profile is then obtained as

$\mathbf{v}(y)=\mathbf{J}(y) / \rho(y)$.

\section{Adiabatic construction}

The adiabatic system is defined as having the same equilibrium one-body density as the nonequilibrium system [31]. The Mermin-Evans theorem, which is at the heart of DFT, states that in equilibrium for a system with given internal interaction potential, at temperature $T$, volume $V$ and chemical potential $\mu$ fixed, there is a unique 
mapping from a given one-body density distribution $\rho(\mathbf{r})$ to a corresponding external potential $[26,27]$. In order to construct the adiabatic system, we use two different methods which we show below to give consistent results.

The adiabatic construction implies to take the nonequilibrium density as an equilibrium density and to calculate the corresponding external potential $V_{\mathrm{ad}}(y)=$ $-\mu_{\mathrm{ad}}(y)$, where $\mu_{\mathrm{ad}}$ denotes the intrinsic chemical potential in the adiabatic system. We use the scaled-particle theory (SPT) equation of state for the hard disk fluid [27], which implies the following form of the chemical potential:

$\frac{\mu_{\mathrm{ad}}^{\mathrm{SPT}}}{k_{B} T}=\ln \left(\frac{\rho \Lambda^{3}}{1-\eta^{*}}\right)+\frac{\left(3-2 \eta^{*}\right) \eta^{*}}{\left(1-\eta^{*}\right)^{2}}$.

Here, $\eta^{*}=\pi / 4 \sigma_{\mathrm{BH}}^{2} \rho$, where $\sigma_{\mathrm{BH}}$ denotes the BarkerHenderson diameter [27], given by $\sigma_{\mathrm{BH}}=\int_{0}^{\infty} d r(1-$ $\left.e^{-\phi(r) /\left(k_{B} T\right)}\right)$. The thermal wavelength $\Lambda$ only adds an irrelevant constant to the chemical potential. Using Eq. 18 is the first way we construct the adiabatic system. The notation $V_{\mathrm{ad}}^{\mathrm{SPT}}(y)$ is used to denote external potentials calculated this way. Note that the dependence of the right hand side of Eq. 18 on $y$ is explicitly known, as it arises from the known density profile $\rho(y)$, taken as an input.

The second way of performing the adiabatic construction also employs an approach based on a local density approximation (LDA), but using simulation data as input. Using the LDA is reasonable for the cases considered in the results section below, as the density profile $\rho$ varies slowly on the scale of the particle size $\sigma$. For given nonequilibrium conditions, we base the LDA on simulation data of an equilibrium system (i.e., for $\dot{\gamma}=0$ ) with an unchanged value of $V_{0}^{\text {ext }}$ as compared to the nonequilibrium system. This yields, in an LDA approximation, the bulk chemical potential of the system as a function of density, as both the (imposed) external potential $V_{\text {ext }}(y)$ and the (sampled) density profile $\rho_{\mathrm{eq}}(y)$ are known as a function of position $y$. Eliminating the parameter $y$ then yields the desired form of the chemical potential as a function of density. In more detail, let $y_{\mathrm{eq}}(\rho)$ be the inverse function of $\rho_{\mathrm{eq}}(y)$. We then obtain the (bulk) chemical potential as a function of (bulk) density as

$\mu_{\mathrm{ad}}^{\mathrm{LDA}}(\rho)=-V_{\mathrm{ext}}\left(y_{\mathrm{eq}}(\rho)\right)$.

Using the equation of state Eq. 19 and the results for the nonequilibrium density profile $\rho(y)$, we can now calculate the external potential in the adiabatic system via $V_{\mathrm{ad}}^{\mathrm{LDA}}(y)=$ $-\mu_{\mathrm{ad}}^{\mathrm{LDA}}(\rho(y))$.

\section{Brownian dynamics simulations}

To simulate the system described in "Description of the system," Brownian dynamics simulations are used. These are based on the integration of Eq. 7, which can be performed with the Euler algorithm,

$\mathbf{r}_{i}(t+\Delta t)=\mathbf{r}_{i}(t)+\xi^{-1}\left(\mathbf{f}_{i}^{\text {int }}\left(\mathbf{r}^{N}\right)+\mathbf{f}_{\mathrm{ext}}\left(y_{i}\right)\right) \Delta t+\delta \mathbf{r}_{i}$.

Here, $\Delta t$ is the time step used in the algorithm and $\delta \mathbf{r}_{i}$ is a random displacement with Gaussian distribution of standard deviation $\sqrt{2 \Delta t k_{B} T / \xi}$. We use the BoxMuller transformation to generate the Gaussian distribution from random numbers generated by the Mersenne Twister algorithm.

At the start of each simulation run, $N$ particles are randomly distributed in a square simulation box of size $V_{2 \mathrm{D}}=N \pi \sigma^{2} / \eta$, while ensuring that there are no overlaps between any pair of the particles. The side length of the square box is $L=\sqrt{V_{2 \mathrm{D}}}$. We use Lees-Edwards (sliding box) boundary conditions [58, 59].

All our simulations were performed with $N=256$ particles and with a time step of $\Delta t=5 \cdot 10^{-6} \tau_{0}$ where $\tau_{0}$ denotes the reduced time scale $\tau_{0}=\xi \sigma^{2} / \epsilon$. The constants $\epsilon, \xi$, and $\sigma$ are set to unity; we only consider the case of the reduced temperature $T^{*}=k_{B} T / \epsilon=1$. The packing fraction $\eta$, the shear rate $\dot{\gamma}$, and strength of the confinement $V_{0}^{\text {ext }}$ are our control parameters. When the Peclet number $\mathrm{Pe}$ is defined using the particle diameter (rather than the particle radius) as the unit of length, the value of our dimensionless shear rate is identical to that of the Peclet number, as $\mathrm{Pe} \equiv \dot{\gamma} \sigma^{2} / D=\dot{\gamma} \tau_{0} / T^{*}$, where the diffusion constant is defined as $D=k_{B} T / \xi$.

The one-body density $\rho(y)$ and the average instantaneous force due to interparticle interaction $\mathbf{f}_{\text {int }}(y)$ are sampled. The histograms used for the sampling of these parameters have a resolution of $\sigma / 15$ but are down sampled to a resolution of $\sigma / 5$ to reduce the noise. For each presented set of parameters, multiple simulations were run (typically at least 150). The results of these are then averaged to produce the final data. Each of the simulations is at first run for $t_{\mathrm{eq}}=0.75 \tau_{0}$, so the system can relax into the steady state. After $t_{\mathrm{eq}}$ has passed, the sampling of the physical quantities of interest is started at a rate of $2000 \tau_{0}^{-1}$. The simulation is then kept running for $t_{\text {run }}=200 \tau_{0}$.

In order to test whether the steady state is reached after $t_{\text {eq }}$, four sets of parameters have additionally been run with $t_{\mathrm{eq}}=100 \tau_{0}$ and $t_{\text {run }}=100 \tau_{0}$ and were then compared to the results of the density profiles produced by simulations with $t_{\text {eq }}$ and $t_{\text {run }}$. The results of these runs show that the difference between the datasets is smaller than $1 \%$ and 
shows no bias, indicating that $t_{\mathrm{eq}}=0.75 \tau_{0}$ is sufficient for the relaxation into the steady state. For smaller values of the shear rate than considered here, using longer relaxation times $t_{\text {eq }}$ might be necessary.

\section{Results}

\section{Behavior of the density profile}

We first present the effects that varying the shear rate $\dot{\gamma}$ and the strength of the compression $V_{0}^{\text {ext }}$ has on the density profile $\rho(y)$ of the system. We have considered moderate values of packing fraction ranging from $\eta=0.1$ to 0.35 . As a reference, the hard disk fluid transitions to a glassy state for packing fractions larger than $\eta=0.699$ [60]. In equilibrium, the hard disk fluid is stable for packing fraction below $0.701 \equiv(\pi / 4) 0.892$ (cf. Ref. [61]). The presence of the confining potential leads to a local increase in density, which potentially could increase the local density above the transition packing fraction for higher values of $\eta$. As different sets of the parameters only appear to lead to quantitative changes of the density profile and not to different physical effects, results are presented for selected values of parameters.

In Fig. 2, results of simulations with packing fractions of 0.2 and 0.3 are presented. Starting with $\eta=0.2$ in panel (a), we show the effects of an increase in the strength of the confinement on the density profile, while the shear rate is kept constant. We observe that the density is compressed into the trough of the conservative potential $V_{\text {ext }}$ and that an increase of $V_{0}^{\text {ext }}$ leads to a corresponding increase in the amplitude of the peak in the density profile. Figure $2 \mathrm{c}$ shows how the density profile at constant strength of confinement is altered by the shear rate. It is apparent that shearing the system leads to a pronounced flattening of the density profile. Figure $2 b$ and $d$ are shown to evaluate the effect that a change in packing fraction has on the density profiles. One can observe that the magnitude of the deviation of the local density from the bulk density $\rho_{\mathrm{b}}=N / V_{2 \mathrm{D}}$ for corresponding sets of parameters remains similar.

density profiles

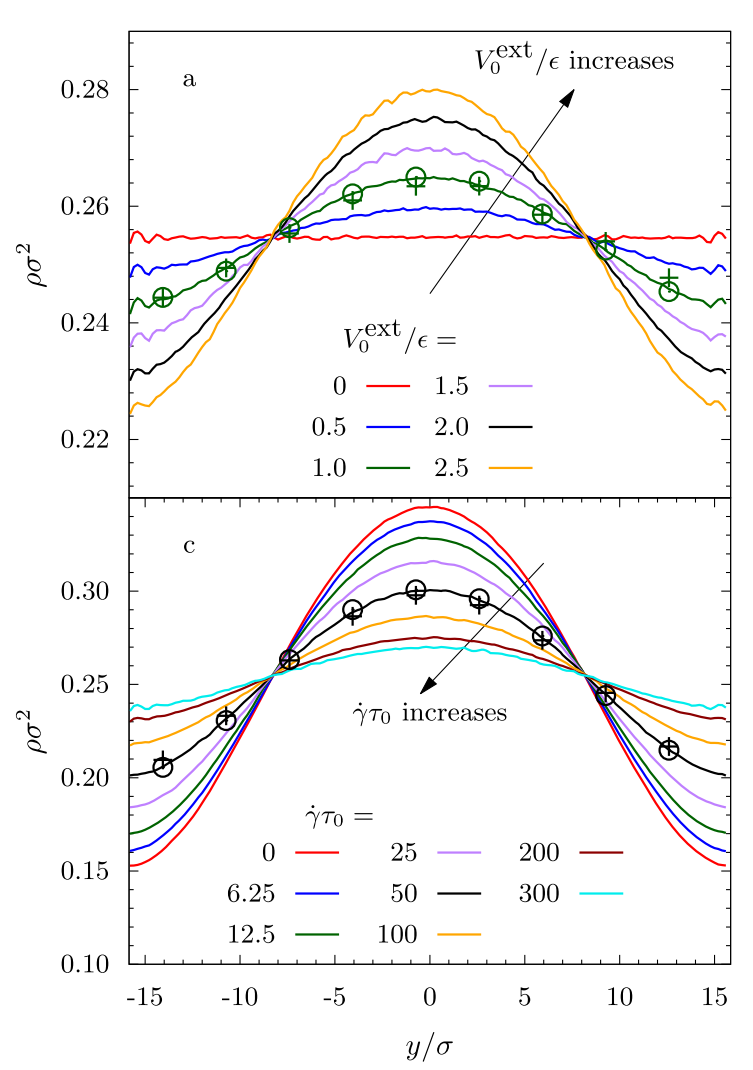

Fig. 2 Density profiles $\rho(y)$ as a function of $y / \sigma$ for various sets of parameters $\eta, \dot{\gamma}$, and $V_{0}^{\text {ext }}$ obtained from BD simulations (lines). In each panel, two of the parameters are kept constant while varying the third (as indicated). Panel a: $\eta=0.2, \dot{\gamma} \tau_{0}=200$. Panel $\mathrm{b}: \eta=0.3, \dot{\gamma} \tau_{0}=100$. Panel c: $\eta=0.2, V_{0}^{\text {ext }} / \epsilon=2$. Panel d:

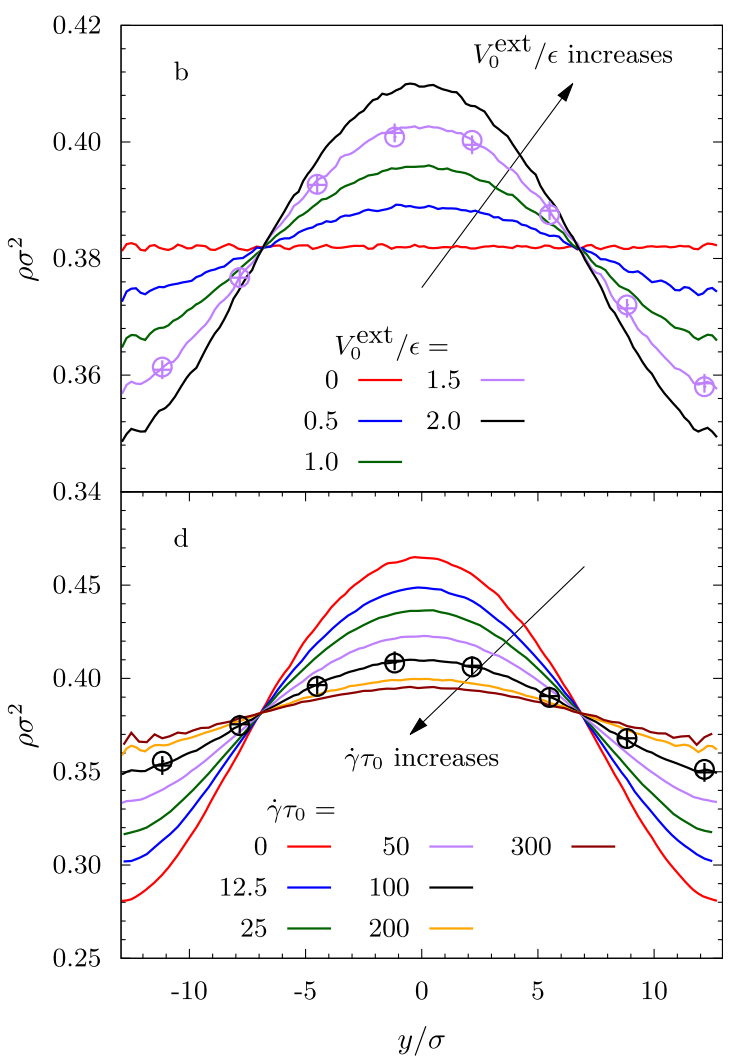

$\eta=0.3, V_{0}^{\text {ext }} / \epsilon=2$. The effect of variation of a single parameter on the density profile is indicated by an arrow. The symbols denote density profiles $\rho_{\mathrm{ad}}^{\mathrm{LDA}}(y)$ (crosses) and $\rho_{\mathrm{ad}}^{\mathrm{SPT}}(y)$ (circles), obtained by the two methods of adiabatic construction described in Section "Adiabatic construction" 


\section{Forces in the nonequilibrium system}

We next present results for the internal force field $\mathbf{f}_{\text {int }}(y)$. This is a particularly interesting quantity, as it contains the superadiabatic part of the force in the nonequilibrium system (cf. Eq. 1). We first investigate the $y$-component, $f_{y}^{\text {int }}$ (which acts in the gradient direction). The main panels in Fig. 3 display the results for the same control parameters as the density profiles shown in Fig. 2. Figure $3 a$ and $b$ illustrate the relationship between the degree of confinement of the system and the behavior of the internal force field, in steady state. As expected, Fig. 3a shows that the magnitude of $f_{y}^{\text {int }}$ increases as the applied external potential is increased. One can also observe that in the homogeneous sheared system (i.e., without compression, $V_{0}^{\text {ext }}=0$ and therefore with constant density profile), $f_{y}^{\text {int }}$ vanishes. The effects that an increase in shear rate has on $f_{y}^{\text {int }}$ are presented in Fig. $3 \mathrm{c}$ and d. The data shown suggests that the shear rate increases the magnitude of $f_{y}^{\text {int }}$ very rapidly for low shear rates, but $f_{y}^{\text {int }}$ appears to reach a saturated state for $\dot{\gamma} \tau_{0} \rightarrow \infty$. This is intuitively clear considering that $f_{y}^{\text {int }}$ is unlikely to be larger then $-f_{y}^{\text {ext }}$ in steady state. Recall that $V_{\text {ext }}$ is kept constant in Fig. $3 \mathrm{c}$ and d.

The insets in Fig. 3 show the $y$-components of the force fields decomposed as in Eq. 14, along with the total force field $f_{y}^{\text {total }}$, i.e., the sum (14) divided by the local density. We show results for four different systems. We expect $f_{y}^{\text {total }}(y)$ to vanish because of the symmetries in the system. As throughout, subscripts $x$ and $y$ denote the components of vectors. In Fig. 3 e, $\mathrm{f}, \mathrm{g}$ and $\mathrm{h}$, it is apparent that $f_{y}^{\text {total }}$ indeed vanishes identically, apart from some residual noise in the data. The figure also demonstrates that both $f_{y}^{\text {int }}$ and $f_{y}^{\text {ran }}$ act in the opposite direction to $f_{y}^{\text {ext }}(y)$ and their sum exactly cancels the applied compression.

Comparing the results of the equilibrium system shown in Fig. $3 \mathrm{~h}$ to the sheared system in Fig. 3f reveals a pronounced difference in the way the force, due to the compression $f_{y}^{\text {ext }}$, is compensated. In Fig. $3 \mathrm{~h}, f_{y}^{\text {int }}$ is not too different in magnitude to $f_{y}^{\text {ran }}$, while in the nonequilibrium case presented in Fig. 3f, the entropic force $f_{y}^{\text {ran }}$ is much smaller and $f_{y}^{\text {int }}$ almost solely compensates $f_{y}^{\text {ext }}$. This is in line with the results from the density profiles, as the homogenization unavoidably leads to the

gradient direction

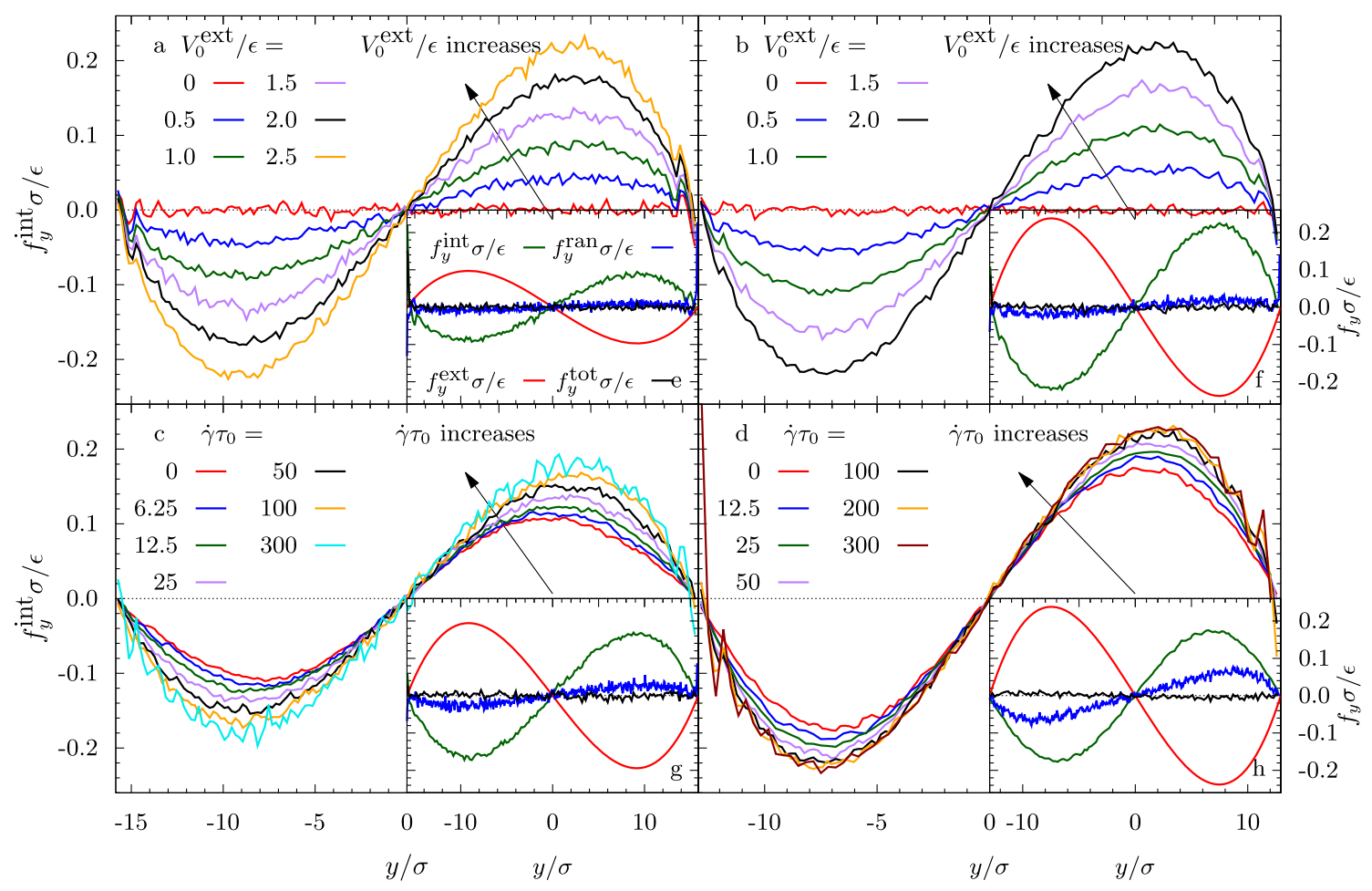

Fig. 3 Internal force field $f_{y}^{\text {int }}(y)$ as a function of $y / \sigma$ for multiple sets of parameters $\eta, \dot{\gamma}$, and $V_{0}^{\text {ext }}$. The parameters held constant in panels a-d are the same as in Fig. 2. The insets show contributions to the force balance in Eq. 14 for four examples. The internal and the total force are sampled in simulations, while the deterministic external force is imposed and the random force is calculated using Eq. 16 and simulation data. Panel e: $\eta=0.2$, $\dot{\gamma} \tau_{0}=200, V_{0}^{\text {ext }} / \epsilon=1$. Panel f: $\eta=0.3, \dot{\gamma} \tau_{0}=100$, $V_{0}^{\text {ext }} / \epsilon=2$. Panel g: $\eta=0.2, \dot{\gamma} \tau_{0}=100, V_{0}^{\text {ext }} / \epsilon=2$. Panel h: $\eta=0.3, \dot{\gamma} \tau_{0}=0, V_{0}^{\text {ext }} / \epsilon=2$ 
entropic force becoming much smaller. Obviously, this effect becomes more apparent as the shear rate and therefore the homogenization is increased, as is clear from a comparison of Fig. 3g and e.

In Fig. 4, the $x$-component of the internal force field, $f_{x}^{\text {int }}$ (i.e., the component that acts in the flow direction) is plotted for the same parameters as $f_{y}^{\text {int }}$ above. We leave out the results for a shear rate of $\dot{\gamma} \tau_{0}=300$, as this is very noisy. We first consider the effects of an increase of $V_{0}^{\text {ext }}$ on the density profile (cf. panels (a) and (b)). One can observe that the relation between $V_{0}^{\text {ext }}$ and $f_{x}^{\text {int }}$ is practically linear. In Fig. $4 \mathrm{c}$ and d, the effects of a variation of the shear rate on $f_{x}^{\text {int }}$ are illustrated. The magnitude of the force increases as the shear rate is increased. However, the force does not increase linearly.

\section{Adiabatic construction}

We use the two methods outlined in "Adiabatic construction" to perform the adiabatic construction. In order to assess their performance, we have carried out simulations of the adiabatic system, i.e., runs in equilibrium under the influence of an external potential $V_{\mathrm{ad}}(y)$, as obtained following the two procedures (SPT and LDA) described in "Adiabatic construction." In the following, we compare the respectively obtained density profiles $\rho_{\mathrm{ad}}^{\mathrm{SPT}}(y)$ and $\rho_{\mathrm{ad}}^{\mathrm{LDA}}(y)$ to each other, as well as to the corresponding (target) nonequilibrium density profile $\rho(y)$.
The adiabatic density profiles for representative parameter choices are shown as symbols in Fig. 2. The quality of the agreement demonstrates that both versions of the adiabatic construction reproduce the density profile of the nonequilibrium systems very well. The relative difference in density is smaller than $\sim 1 \%$. Comparing the different methods of adiabatic construction reveals good agreement between the results, showing that our simulations are able to reproduce the behavior of the hard disk fluid as predicted by SPT. However, the approach using only our simulation data and LDA to calculate the chemical potential is slightly superior. Therefore, we use data obtained with these simulations in the analysis to obtain the superadiabatic force field.

\section{Superadiabatic forces}

Having obtained results for both the internal force field in the nonequilibrium system, $\mathbf{f}_{\text {int }}$, as well as in the adiabatic system, $\mathbf{f}_{\mathrm{ad}}$, we proceed by using the force splitting (1) in order to calculate the superadiabatic forces according to

$\mathbf{f}_{\text {sup }}(y)=\mathbf{f}_{\text {int }}(y)-\mathbf{f}_{\text {ad }}(y)$.

As the adiabatic system is translationally invariant in $x$, we expect the corresponding force component to vanish, $f_{x}^{\text {ad }} \equiv 0$. Our simulation data confirms this expectation. Equation 21 accordingly implies $f_{x}^{\text {sup }}(y)=f_{x}^{\text {int }}(y)$; hence, in the flow direction, the superadiabatic force is identical

flow direction

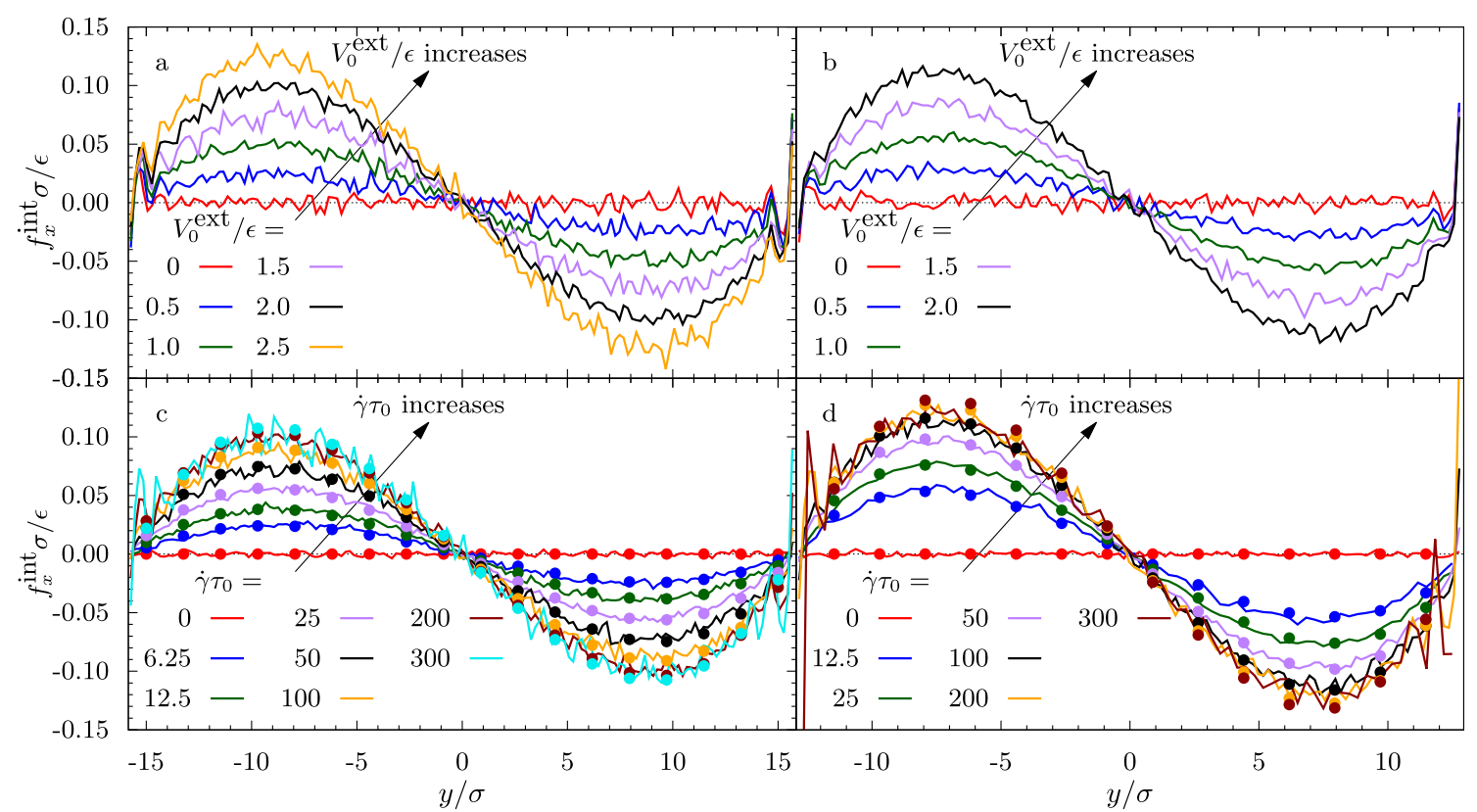

Fig. 4 Viscous force field, scaled as $f_{x}^{\text {int }}(y) \sigma / \epsilon$, as a function of the scaled position coordinate $y / \sigma$ for multiple sets of parameters $\eta, \dot{\gamma}$, and $V_{0}^{\text {ext }}$. The parameters held constant are the same as in Fig. 2. In panels (c) and (d), the circles denote the superadiabatic force calculated using Eq. 22 and the density profiles shown in Fig. 2 
to the full internal force field. These results were discussed above in Section "Forces in the nonequilibrium system".

The situation in the gradient direction is different, as the density inhomogeneity in $y$ leads to $f_{y}^{\text {ad }} \neq 0$. In Fig. 5, the $y$-component of the superadiabatic force field is presented in the main panels. The superadiabatic force behaves in a similar manner as the internal force: It reaches a saturated state as the shear rate $\dot{\gamma}$ is increased and it is proportional to the strength of the external confinement.

Comparing the typical magnitude of the superadiabatic force (shown in Fig. 5) to that of the total internal force (shown in Fig. 3) reveals that even for small shear rates $f_{y}^{\text {sup }}$ already makes up about one-tenth of $f_{y}^{\text {int }}$. The relative contribution of $f_{y}^{\text {sup }}$ increases as the shear rate is increased and surpasses the adiabatic contribution for the intermediate values of shear rate considered. For the highest values of $\dot{\gamma} \tau_{0}$ presented, the internal force is completely dominated by superadiabatic contributions. We conclude that the superadiabatic effects in sheared systems play a significant role and that any attempt at describing the nonequilibrium dynamics requires an understanding of the superadiabatic contribution.

The saturated state corresponds to the state of the system with vanishing density gradient. Comparing both components of $\mathbf{f}_{\text {sup }}(y)$ with each other reveals they have a similar shape, but with the sign flipped and that the $x$ component has a larger magnitude. This again implies a direct link to the density gradient, which we investigate in the following section.

\section{Scaling of the superadiabatic force fields}

The observations described in "Superadiabatic forces" suggest that the superadiabatic force profile depends linearly on the gradient of the density. It is furthermore apparent that this force also depends on the applied shear rate. Assuming a linear dependence on the shear rate, the simplest possible representation of the superadiabatic force as a scaling law is

$f_{y}^{\text {sup }}(y)=c_{y} \dot{\gamma} \nabla \rho(y)$

where the constant $c_{y}$ is dependent on the packing fraction $\eta$ and on $V_{0}^{\text {ext }}$. In order to compare this empirical rule to our data, we need to take the gradient of the densities presented in Fig. 2. As direct numerical differentiation produces very noisy results, we first fit a polynomial of order 4 to the density profiles and then carry out the differentiation analytically.

The results of this calculation (for the $y$-component) are denoted by the circles in Fig. $5 \mathrm{a}_{1}$ and $b_{1}$. We find that for low shear rates up to $\approx 100 \dot{\gamma} \tau_{0}$, the $y$-component of the scaling law shows very good agreement with our data for both considered values of the packing fractions. However, the scaling form overestimates the superadiabatic force in case of high shear rates, which implies that the saturation effects discussed above are not captured perfectly.

An alternative way of testing the form Eq. 22 can be obtained by considering both the force balance in the nonequilibrium system,

$-V_{\mathrm{ext}}^{\prime}(y)+f_{y}^{\mathrm{ad}}(y)+f_{y}^{\mathrm{sup}}(y)-k_{B} T \rho^{\prime}(y) / \rho(y)=0$,

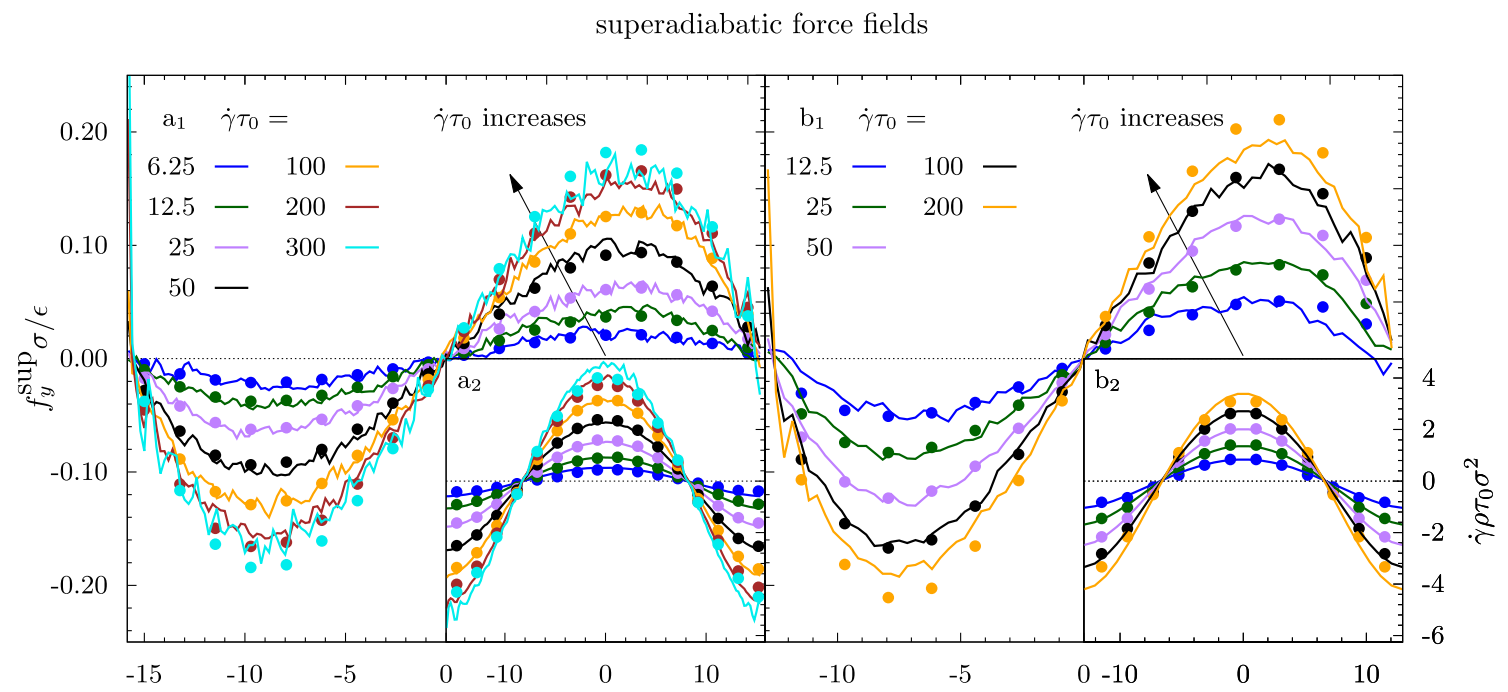

Fig. 5 Panels $\left(\mathrm{a}_{1}\right)$ and $\left(\mathrm{b}_{1}\right)$ : Superadiabatic force field $f_{y}^{\text {sup }}$ for multiple systems. Solid lines denote data obtained in simulations; also shown are the results of the empirical scaling law (Eq. 26). Panels $\left(a_{2}\right)$ and $\left(b_{2}\right)$ : Comparison of the left hand side (solid lines) and the right hand side (circles) of Eq. 25 for the same parameters as in the

main panels. Here, the integration constant $d$ was chosen such that the graphs are centered around $\dot{\gamma} \rho=0$. In panels $\left(\mathrm{a}_{1}\right)$ and $\left(\mathrm{a}_{2}\right)$, the parameters $\eta=0.2$ and $V_{0}^{\text {ext }} / \epsilon=2.0$ are held constant, while in panels $\left(\mathrm{b}_{1}\right)$ and $\left(\mathrm{b}_{2}\right)$, the parameters $\eta=0.3$ and $V_{0}^{\text {ext }} / \epsilon=2.0$ are held constant 
and the force balance in the adiabatic system,

$-V_{\mathrm{ad}}^{\prime}(y)+f_{y}^{\mathrm{ad}}(y)-k_{B} T \rho^{\prime}(y) / \rho(y)=0$,

where the prime denotes the derivative with respect to $y$. Here, Eq. 23 is obtained by dividing the force density balance (13) by the density profile and using the adiabaticsuperadiabatic splitting (1) to express the internal force field $\mathbf{f}_{\text {int }}$ as a sum of adiabatic and superadiabatic force contributions. Observing that the $y$-component of the current vanishes in the sheared steady state then yields (23). Equation 24 is the analogue in the adiabatic system; as the adiabatic state is in equilibrium, there are neither flow nor superadiabatic contributions by construction.

Using Eq. 24 to eliminate $f_{y}^{\text {ad }}$ from Eq. 23, using the scaling form Eq. 22, and integrating in $y$, one arrives at

$\dot{\gamma} \rho(y)=\frac{V_{\mathrm{ad}}(y)-V_{\mathrm{ext}}(y)-d}{c_{y}}$,

where $d$ is an integration constant and $c_{y}$ is the same constant as used in Eq. 22. This balance equation only needs the nonequilibrium density and the adiabatic potential (as obtained in the simulations) as input to carry out a test of the scaling law for the superadiabatic forces. The corresponding results are depicted in panels $a_{2}$ and $b_{2}$ of Fig. 5. One observes the same level of agreement as in the case of the direct comparison of the superadiabatic forces and the scaling law.

In case of the viscous force, we use a form with a modified (empirical) exponent,

$f_{x}^{\text {sup }}(y)=c_{x} \dot{\gamma}^{0.8} \nabla \rho(y)$,

where $c_{x}$ is a fit parameter, which again depends on the values of $\eta$ and of $V_{0}^{\text {ext }}$. In Fig. $4 c$ and d, the results of this effective scaling law are compared to the simulation data of the superadiabatic force. We find again very good agreement with the real data. Similarly to the viscous force, the structural force, the structural force is also overestimated in case of high shear rates close to the saturated state. Although we have not performed a systematic error analysis, we are confident that our data is much more consistent with the unusual value 0.8 of the exponent in Eq. 26 than with an exponent of 1 , or even 0.9. We expect the power law (26) to be an empirical representation of the data rather than a fundamental relationship. We leave the construction of a corresponding approximation for the superadiabatic excess power functional, which is the generator of the nonequilibrium forces via $\mathbf{f}_{\text {sup }}(\mathbf{r}, t)=$ $-\delta P_{t}^{\operatorname{exc}} / \delta \mathbf{J}(\mathbf{r}, t)$, to future work.

\section{Conclusions}

We have systematically investigated the effects that shearing has on the density and force profiles of a two-dimensional system of quasi-hard disks upon which an additional conservative confining force field is applied in the direction normal to the flow. Our results show that the shear flow induces a superadiabatic structural force that acts against the compression of the system. As a result, the density profile approaches the homogeneous bulk density for high shear rates. We have investigated the forces that act in the system. There occurs a dissipative effect from the forces acting against the flow of the particles as well as a structural force. The adiabatic construction was used to identify these superadiabatic force contributions. The concept of superadiabatic forces [31, 32, 44] allows systematic classification [42] of flow and structural effects, including viscous [39-41] and structural [41-43] force contributions.

Future work could be directed at the behavior at low shear rates. Low shear rates are interesting as our current scaling law is not invariant if the shear direction changes, i.e., our scaling law predicts a stronger confinement for such situations. Additionally by investigation of a wider range of parameters, a more definite understanding of the scaling laws could be achieved. Furthermore, considering confining potentials that are less smooth than the one considered here should be interesting, as would be investigating the laning instability reported in Ref. [62], disorder-order transitions reported for three-dimensional systems in Ref. [63] and the general framework for viscosity of Ref. [64]. The results that we provide could form material for an in-depth comparison of PFT and MCT, possibly along the lines of Ref. [33]. Dispersions of thermoresponsive colloids [320] could be excellent model systems for corresponding experimental work that is aimed at systematically studying superadiabatic forces.

Acknowledgment Open Access funding provided by Projekt DEAL. We thank Sophie Hermann and Daniel de las Heras for useful discussions.

Funding Information This work is funded by the Deutsche Forschungsgemeinschaft (DFG, German Research foundation) 317849184 .

\section{Compliance with ethical standards}

Conflict of interests The authors declare that they have no conflict of interest.

Open Access This article is licensed under a Creative Commons Attribution 4.0 International License, which permits use, sharing, adaptation, distribution and reproduction in any medium or format, as long as you give appropriate credit to the original author(s) and the source, provide a link to the Creative Commons licence, and indicate 
if changes were made. The images or other third party material in this article are included in the article's Creative Commons licence, unless indicated otherwise in a credit line to the material. If material is not included in the article's Creative Commons licence and your intended use is not permitted by statutory regulation or exceeds the permitted use, you will need to obtain permission directly from the copyright holder. To view a copy of this licence, visit http:// creativecommonshorg/licenses/by/4.0/.

\section{References}

1. Brader JM (2010) Nonlinear rheology of colloidal dispersions. J Phys Condens Matter 22:363101

2. Dhont JKG (1996) An introduction to the dynamics of colloids. Elsevier, Amsterdam

3. Senff H, Richtering W, Norhausen C, Weiss A, Ballauff M (1999) Rheology of a temperature sensitive core-shell latex. Langmuir 15:102

4. Deike I, Ballauff M (2001) Rheology of thermosensitive latex particles including the high-frequency limit. J Rheo 45:709

5. Fuchs M, Ballauff M (2005) Flow curves of dense colloidal dispersions: schematic model analysis of the shear-dependent viscosity near the colloidal glass transition. J Chem Phys 122:094707

6. Fuchs M, Ballauff M (2005) Nonlinear rheology of dense colloidal dispersions: a phenomenological model and its connection to mode coupling theory. Col Surf A 270:232

7. Crassous JJ, Siebenbürger M, Ballauff M, Drechsler M, Henrich O, Fuchs M (2006) Thermosensitive core-shell particles as model systems for studying the flow behavior of concentrated colloidal dispersions. J Chem Phys 125:204906

8. Crassous JJ, Siebenbürger M, Ballauff M, Drechsler M, Hajnal D, Henrich O, Fuchs M (2008) Shear stresses of colloidal dispersions at the glass transition in equilibrium and in flow. J Chem Phys 128:204902

9. Siebenbürger M, Fuchs M, Winter HH, Ballauff M (2009) Viscoelasticity and shear flow of concentrated, noncrystallizing colloidal suspensions: comparison with mode-coupling theory. J Rheol 53:707

10. Winter HH, Siebenbürger M, Hajnal D, Henrich O, Fuchs M, Ballauff M (2009) An empirical constitutive law for concentrated colloidal suspensions in the approach of the glass transition. Rheo Acta 48:747

11. Brader JM, Siebenbürger M, Ballauff M, Reinheimer K, Wilhelm M, Frey SJ, Weysser F, Fuchs M (2010) Nonlinear response of dense colloidal suspensions under oscillatory shear: modecoupling theory and Fourier transform rheology experiments. Phys Rev E 82:061401

12. Siebenbürger M, Fuchs M, Ballauff M (2012) Core-shell microgels as model colloids for rheological studies. Soft Matter 8:4014

13. Siebenbürger M, Ballauff M, Voigtmann T (2012) Creep in colloidal glasses. Phys Rev Lett 108:255701

14. Chu FF, Siebenbürger M, Polzer F, Stolze C, Kaiser J, Hoffmann M, Heptner N, Dzubiella J, Drechsler M, Lu Y, Ballauff M (2012) Synthesis and characterization of monodisperse thermosensitive dumbbell-shaped microgels. Macromolec Rap Comm 33:1042

15. Amann CP, Siebenbürger M, Krüger M, Weysser F, Ballauff M, Fuchs M (2013) Overshoots in stress-strain curves: colloid experiments and schematic mode coupling theory. J Rheol 57:149

16. Ballauff M, Brader JM, Egelhaaf SU, Fuchs M, Horbach J, Koumakis N, Krüger M, Laurati M, Mutch KJ, Petekidis G,
Siebenbürger M, Voigtmann T, Zausch J (2013) Residual stresses in glasses. Phys Rev Lett 110:215701

17. Amann CM, Siebenbürger M, Ballauff M, Fuchs M (2015) Nonlinear rheology of glass-forming colloidal dispersions: transient stress-strain relations from anisotropic mode coupling theory and thermosensitive microgels. J Phys: Condensed Matt 27:194121

18. Chu FF, Heptner N, Lu Y, Siebenbürger M, Lindner P, Dzubiella J, Ballauff M (2015) Colloidal plastic crystals in a shear field. Langmuir 31:5992

19. Heptner N, Chu FF, Lu Y, Lindner P, Ballauff M, Dzubiella J (2015) Nonequilibrium structure of colloidal dumbbells under oscillatory shear. Phys Rev E 92:052311

20. Seyboldt R, Merger D, Coupette F, Siebenbürger M, Ballauff M, Wilhelm M, Fuchs M (2016) Divergence of the third harmonic stress response to oscillatory strain approaching the glass transition. Soft Matter 12:8825

21. Brader JM, Krüger M (2011) Density profiles of a colloidal liquid at a wall under shear flow. Mol Phys 109:1029

22. Krüger M, Brader JM (2011) Controlling colloidal sedimentation using time-dependent shear. EPL 96:68006

23. Scacchi A, Krüger M, Brader JM (2016) Driven colloidal fluids: construction of dynamical density functional theories from exactly solvable limits. J Phys: Condens Matter 28:244023

24. Aerov AA, Krüger M (2014) Driven colloidal suspensions in confinement and density functional theory: microstructure and wall-slip. J Chem Phys 140:094701

25. Aerov AA, Krüger M (2015) Theory of rheology in confinement. Phys Rev E 92:042301

26. Evans R (1979) The nature of the liquid-vapour interface and other topics in the statistical mechanics of non-uniform, classical fluids. Adv Phys 28:143

27. Hansen J-P, McDonald IR Theory of simple liquids, Academic Press, London 2013, 4th ed

28. Archer AJ, Chacko B, Evans R (2017) The standard mean-field treatment of inter-particle attraction in classical DFT is better than one might expect. J Chem Phys 147:034501

29. Marconi UMB, Tarazona P (1999) Dynamic density functional theory of fluids. J Chem Phys 110:8032

30. Archer AJ, Evans R (2004) Dynamical density functional theory and its application to spinodal decomposition. J Chem Phys 121:4254

31. Schmidt M, Brader JM (2013) Power functional theory for Brownian dynamics. J Chem Phys 138:214101

32. Fortini A, de las Heras D, Brader JM, Schmidt M (2014) Superadiabatic forces in Brownian many-body dynamics. Phys Rev Lett 113:167801

33. Brader JM, Schmidt M (2013) Nonequilibrium Ornstein-Zernike relation for Brownian many-body dynamics. J Chem Phys 139:104108

34. Brader JM, Schmidt M (2014) Dynamic correlations in Brownian many-body systems. J Chem Phys 140:034104

35. Brader JM, Schmidt M (2015) Power functional theory for the dynamic test particle limit. J Phys: Condens Matter 27:194106

36. Schindler T, Schmidt M (2016) Dynamic pair correlations and superadiabatic forces in a dense Brownian liquid. J Chem Phys 145:064506

37. Treffenstädt LL, Schmidt M (2020) Dynamical universality in liquids. to be published

38. Brader JM, Schmidt M (2015) Free power dissipation from functional line integration. Mol Phys 113:2873

39. de las Heras D, Schmidt M (2018) Velocity gradient power functional for Brownian dynamics. Phys Rev Lett 120:028001

40. Treffenstädt LL, Schmidt M (2020) Memory-induced motion reversal in Brownian liquids. Soft Matter 16:1518 
41. Stuhlmüller NCX, Eckert T, de las Heras D, Schmidt M (2018) Structural nonequilibrium forces in driven colloidal systems. Phys Rev Lett 121:098002

42. de las Heras D, Schmidt M (2020) Flow and structure in nonequilibrium Brownian many-body systems. to be published

43. Geigenfeind T, de las Heras D, Schmidt M (2020) Superadiabatic demixing in nonequilibrium colloids. Comms Phys 3:23

44. Bernreuther E, Schmidt M (2016) Superadiabatic forces in the dynamics of the one-dimensional Gaussian core model. Phys Rev E 94:022105

45. de las Heras D, Renner J, Schmidt M (2019) Custom flow in overdamped Brownian dynamics. Phys Rev E 99:023306

46. Krinninger P, Schmidt M, Brader JM (2016) Nonequilibrium phase behaviour from minimization of free power dissipation. Phys Rev Lett 117:208003. Erratum, 119, 029902 (2017)

47. Krinninger P, Schmidt M (2019) Power functional theory for active Brownian particles: general formulation and power sum rules. J Chem Phys 074112:150

48. Hermann S, Krinninger P, de las Heras D, Schmidt M (2019) Phase coexistence of active Brownian particles. Phys Rev E 100:052604

49. Hermann S, de las Heras D, Schmidt M (2019) Non-negative interfacial tension in phase-separated active Brownian particles. Phys Rev Lett 128:268002

50. Hermann S, Schmidt M Active interface polarization is a state function. Phys. Rev. Research (Rap. Comm., 2020, to be published)

51. Schmidt M (2018) Power functional theory for Newtonian manybody dynamics. J Chem Phys 148:044502

52. Schmidt M (2015) Quantum power functional theory for manybody dynamics. J Chem Phys 143:174108

53. Brütting M, Trepl T, de las Heras D, Schmidt M (2019) Superadiabatic forces via the acceleration gradient in quantum many-body dynamics. Molecules 24:3660

54. de las Heras D, Schmidt M (2018) Better than counting: density profiles from force sampling. Phys Rev Lett 120:218001

55. Hermann S, Schmidt M (2018) Active ideal sedimentation: exact two-dimensional steady states. Soft Matter 14:1614

56. de las Heras D, Schmidt M (2014) Full canonical information from grand potential density functional theory. Phys Rev Lett 113:238304

57. de las Heras D, Brader JM, Fortini A, Schmidt M (2016) Particle conservation in dynamical density functional theory. J Phys Condens Matter 28:244024

58. Edwards SF, Lees AW (1972) The computer study of transport processes under extreme conditions. J Phys C 5:1921

59. Allen MP (1989) Computer simulation of liquids. Clarendon Press, Oxford. revised Ed.

60. Piasecki J, Szymczak P, Kozak JJ (2010) Prediction of a structural transition in the hard disk fluid. J Chem Phys 133:164507

61. Kapfer SC, Krauth W (2015) Two-dimensional melting: from liquid-hexatic coexistence to continuous transitions. Phys Rev Lett 114:035702
62. Scacchi A, Archer AJ, Brader JM (2017) Dynamical density functional theory analysis of the laning instability in sheared soft matter. Phys Rev E 96:062616

63. Foss DR, Brady JF (2000) Brownian dynamics simulation of hard-sphere colloidal dispersions. J Rheol 44:629

64. Makuch K, Holyst R, Kalwarczyk T, Garstecki P, Brady JF (2020) Diffusion and flow in complex liquids. Soft Matter 16:114

Publisher's note Springer Nature remains neutral with regard to jurisdictional claims in published maps and institutional affiliations.

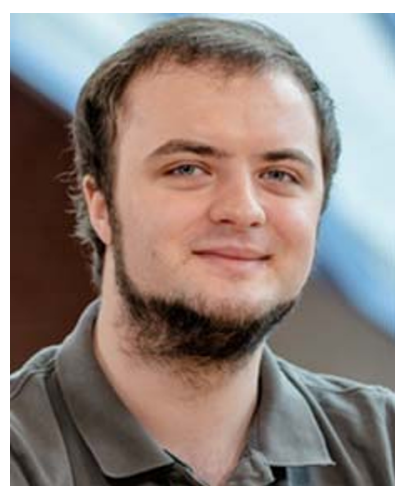

Nikolai Jahreis is a Master student of physics at the University of Bayreuth and an employee of the IT Servicecenter of the UBT. He was the students' representative (Vorsitzender der Fachschaft MPI) in 2018/2019 and the technical director for teaching video production in the Physics Department.

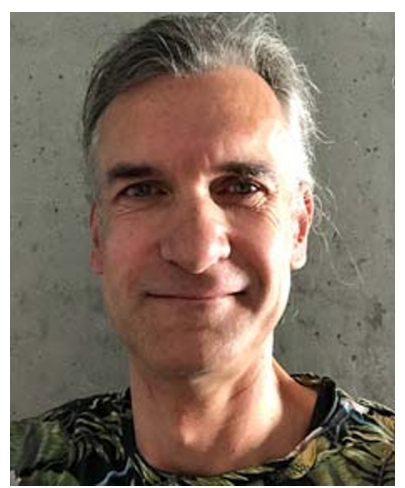

Matthias Schmidt holds the Chair for Theoretical Physics II at the University of Bayreuth. He studied physics in Erlangen and obtained $\mathrm{PhD}$ and Habilitation at the Heinrich-HeineUniversität Düsseldorf. $\mathrm{He}$ was a software developer at Siemens and a postdoc in Fargo and in Utrecht, before becoming a staff member at the University of Bristol in 2005. When arriving at Bayreuth in 2008 , he was warmly welcomed, strategically connected, and scientifically much stimulated by Matthias Ballauff, to whom this contribution is dedicated. 